\title{
FUNÇÃO DE RESPOSTA DA ALFACE AMERICANA AOS NÍVEIS DE ÁGUA E ADUBAÇÃO NITROGENADA ${ }^{1}$
}

\author{
Response function of the crisphead lettuce under irrigation depths and nitrogen rates
}

\author{
Patrícia Aparecida Marques Silva², Geraldo Magela Pereira ${ }^{3}$, Ricardo Pereira Reis ${ }^{4}$, \\ Luís Antônio Lima ${ }^{3}$, José Henrique da Silva Taveira ${ }^{5}$
}

\begin{abstract}
RESUMO
Objetivando-se estudar o efeito das lâminas de água, doses de nitrogênio e sua interação sobre os rendimentos produtivo e econômico da cultura da alface americana irrigada por gotejamento, conduziu-se um experimento em casa-de-vegetação localizada na Universidade Federal de Lavras, Minas Gerais, no período de setembro a novembro de 2004. O delineamento experimental foi fatorial 4 x 4 em blocos casualizados, sendo que os tratamentos constituíram-se da combinação de quatro lâminas de irrigação ( $\mathrm{W}_{1}=91,99$; $\left.\mathrm{W}_{2}=142,79 ; \mathrm{W}_{3}=186,34 ; \mathrm{W}_{4}=237,14 \mathrm{~mm}\right)$ com quatro níveis de adubação nitrogenada $\left(\mathrm{N}_{0}=0 ; \mathrm{N}_{1}=80 ; \mathrm{N}_{2}=160 ; \mathrm{N}_{3}=320 \mathrm{~kg}\right.$ ha $\left.{ }^{-1}\right)$ e com trê̂s repetições. Utilizou-se a cultura da alface americana, cultivar Raider, irrigações diárias controladas com base na evaporação de um minitanque $(\mathrm{EVm})$, sendo os tratamentos $\mathrm{W}_{1}, \mathrm{~W}_{2}, \mathrm{~W}_{3}$ e $\mathrm{W}_{4}$, correspondentes a $0,35 \mathrm{EVm}, 0,70 \mathrm{EVm}, 1,0 \mathrm{EVm}$ e $1,35 \mathrm{EVm}$, respectivamente. A cultura foi instalada no espaçamento de $0,30 \mathrm{~m}$ x $0,30 \mathrm{~m}$, irrigada por um sistema de irrigação por gotejamento, com gotejadores autocompensantes de $1,6 \mathrm{~L} \mathrm{~h}^{-1}$ de vazão, espaçados de $0,3 \mathrm{~m}$. Após a análise dos resultados concluiu-se que a aplicação de 205,26 mm de água e de $257,14 \mathrm{~kg} \mathrm{ha}^{-1}$ de nitrogênio proporcionou estimar uma produtividade máxima de $26.959,93 \mathrm{~kg} \mathrm{ha}^{-1}$ que correspondeu à obtenção do lucro máximo, considerando os preços dos fatores água $\left(R \$ 0,44 \mathrm{~mm}^{-1}\right)$, nitrogênio $\left(\mathrm{R} \$ 2,09 \mathrm{~kg}^{-1}\right) \mathrm{e}$ da alface americana $\left(\mathrm{R} \$ 0,80 \mathrm{~kg}^{-1}\right)$. A produtividade física máxima de $27.004,49 \mathrm{~kg} \mathrm{ha}^{-1}$ foi estimada com $208,03 \mathrm{~mm}$ e $290,5 \mathrm{~kg} \mathrm{ha}^{-1} \mathrm{de}$ nitrogênio. O menor valor médio da produtividade comercial da alface americana $\left(13.814,25 \mathrm{~kg} \mathrm{ha}^{-1}\right)$ foi obtido no tratamento onde foi aplicada a menor lâmina de água $(91,99 \mathrm{~mm})$. Essa produtividade é inferior àquela obtida no tratamento com dose $0 \mathrm{~kg}^{\mathrm{ha}} \mathrm{he}^{-1} \mathrm{de}$ nitrogênio, cuja produtividade média foi de $19.943,7 \mathrm{~kg} \mathrm{ha}^{-1}$.
\end{abstract}

Termos para indexação: Lactuca sativa L., Irrigação por gotejamento, Análise econômica.

\begin{abstract}
The main objective of this experiment carried out from September to December/2004, under greenhouse at the Federal University of Lavras, in Minas Gerais, was to study the effect of water and nitrogen applications rates, and the interaction between these factors on both the yieldand economics of crisphead lettuce. A 4 x 4 factorial experimental design with randomized blocks and three replications was used. Treatments were determined by combinations of four water depths $\left(\mathrm{W}_{1}=91.99 ; \mathrm{W}_{2}=142.79 ; \mathrm{W}_{3}=186.34\right.$; $\left.\mathrm{W}_{4}=237.14 \mathrm{~mm}\right)$ and four nitrogen levels $\left(\mathrm{N}_{0}=0 ; \mathrm{N}_{1}=80 ; \mathrm{N}_{2}=160 ; \mathrm{N}_{3}=320 \mathrm{~kg} \mathrm{ha}^{-1}\right)$. Crisphead lettuce (cv. raider) was grown under daily irrigations applications computed based on the evaporation measured on a reduced pan $(E V m)$. Treatments $\mathrm{W}_{1}, \mathrm{~W}_{2}, \mathrm{~W}_{3} \mathrm{e} \mathrm{W}_{4}$, corresponded to, respectively, $0.35 \mathrm{EVm}, 0.70 \mathrm{EVm}, 1.0 \mathrm{EVm}$, and $1.35 \mathrm{EVm}$. A $0.30 \mathrm{~m}$ x $0.30 \mathrm{~m}$ plant spacing was observed and irrigation water was applied trough a drip irrigation system with $1.6 \mathrm{~L} \mathrm{~h}^{1}$ emitters spaced $0.30 \mathrm{~m}$ apart. Under the cost scenario considered, water at $\mathrm{R} \$ 0.44 \mathrm{~mm}^{-1}$, nitrogen at $\mathrm{R} \$ 2.09 \mathrm{~kg}^{-1}$ and crisphead lettuce at $\mathrm{R} \$ 0.80 \mathrm{~kg}^{-1}$, the maxim profit, and the maxim economical productivity, estimated at $26,960 \mathrm{~kg} \mathrm{ha}^{-1}$ was achieved with the application of $205 \mathrm{~mm}$ of water and $257 \mathrm{~kg} \mathrm{ha}^{-1}$ of nitrogen. The maxim physical productivity $\left(27,004 \mathrm{~kg}^{\prime} \mathrm{ha}^{-1}\right)$ would be achieved by applying $20,803 \mathrm{~mm}$ of water and $290.5 \mathrm{~kg} \mathrm{ha}^{-1}$ of nitrogen. The smaller average value of crisphead lettuce commercial yield $\left(13,814 \mathrm{~kg} \mathrm{ha}^{-1}\right)$ was achieved with the application of the lowest water depth $(91.99 \mathrm{~mm})$ which was smaller than the yield achieved by the treatment with $0 \mathrm{~kg} \mathrm{ha}^{-1}$ of nitrogen $\left(19,944 \mathrm{~kg} \mathrm{ha}^{-1}\right)$.
\end{abstract}

Index terms: Lactuca sativa L., Drip irrigation, Economic analysis.

(Recebido em 3 de abril de 2006 e aprovado em 19 de junho de 2007)

\section{INTRODUÇÃO}

A otimização da rentabilidade constitui o principal objetivo da empresa agrícola, e está associada ao uso racional dos recursos disponíveis no processo de produção de forma a se obterem os mais altos níveis de rendimento econômico. A produção agrícola tem como fatores complementares na rentabilidade econômica a água

\footnotetext{
"Parte da tese apresentada pelo primeiro autor à Universidade Federal de Lavras, para obtenção do título de "Doctor Science" em Engenharia Agrícola. Pesquisa financiada pela Fapemig - Processo № EDT 2232/03.

Engenheira Agrícola, Doutora - Departamento de Engenharia/DEG - Universidade Federal de Lavras/UFLA - Cx.P. 3037 - $37200-000$ - Lavras, MG patsilva@ufla.br

${ }^{3}$ Doutores, Professores Associado, Bolsista do CNPq - Departamento de Engenharia/DEG - Universidade Federal de Lavras/UFLA - Cx.P. 3037 - $37200-000$ Lavras, MG - geraldop@ufla.br; lalima@ufla.br

${ }^{4}$ Doutor, Professor Titular, Bolsista do CNPq - Departamento de Administração e Economia/DAE - Universidade Federal de Lavras/UFLA - Cx.P. 3037 37200-000 - Lavras, MG - ricpreis@uflabr

${ }^{5}$ Mestrando -Departamento de Engenharia/DEG - Universidade Federal de Lavras/UFLA- Cx.P. 3037-37200-000 -Lavras, MG-henriquetaveira@yahoo.com.br
} 
e os nutrientes, fatores preponderantes para o êxito da agricultura. Além disso, as maiores variações no rendimento da cultura em questão é a resposta às mudanças nos níveis desses dois recursos, expressando a alta sensibilidade do rendimento aos níveis desses fatores de produção. Portanto, ao utilizar os procedimentos das funções de resposta permite-se encontrar soluções na otimização do uso da água e dos fertilizantes, obtendo-se o máximo do produto com determinado custo de produção (SOARES et al., 2002).

Segundo Reichardt (1978), a água é indispensável para a produção, e por isso a sua falta ou excesso afeta, significativamente, a produtividade de uma cultura, tornando indispensável o seu manejo racional para se conseguir a maximização da produção. Foi comprovado por diversos autores que a irrigação afeta diretamente a produtividade em culturas como o melão (BEZERRA \& MOURÃO, 2000; MEDEIROS et al., 2000), feijão (PEREIRA et al., 2000), abóbora (MAROUELLI et al., 2000), melancia (ANDRADE JÚNIOR et al., 1996), além da alface crespa (PEREIRA et al., 2003).

$\mathrm{O}$ desenvolvimento da agricultura irrigada entre outros aspectos requer maior eficiência em relação à aplicação de água e nutrientes. Pereira et al. (2000) estudando os efeitos das lâminas de água e doses de nitrogênio em cobertura sobre a produção do feijoeiro (Phaseolus vulgaris L.), observaram que o feijoeiro respondeu positivamente à irrigação, até o nível em que o excesso de água foi prejudicial ao seu desenvolvimento. A produção de grãos aumentou com a elevação da dose de nitrogênio em cobertura. A lâmina de água apresentou efeito significativo para a massa de 100 grãos, número de vagens por planta e de grãos por vagem. O nitrogênio em cobertura foi significativo para massa de 100 grãos, número e massa de grãos por vagem. Marouelli et al. (2000) estudando a resposta da aboboreira a diferentes lâminas de água e doses de nitrogênio, constataram que o número máximo de frutos comerciáveis foi obtido para a dose de nitrogênio de 114 $\mathrm{kg} \mathrm{ha}^{-1} \mathrm{e}$ a lâmina de irrigação foi de $401 \mathrm{~mm}$, enquanto a produtividade máxima foi obtida para $109 \mathrm{~kg} \mathrm{ha}^{-1}$ e $385 \mathrm{~mm}$.

Observa-se que tanto para lâminas de irrigação como para níveis de adubação são recomendações genéricas que objetivam a obtenção de produtividades físicas máximas, podendo ser inviável sob o ponto de vista econômico, já que a eficiência econômica não corresponde necessariamente à eficiência técnica. Pereira et al. (2003) estudando a produção da alface crespa cv. Verônica cultivada em vasos, em função de água e nitrogênio concluiu que a produção de alface em ambiente protegido pode ser realizada com déficit e que nesse caso também varia com o conteúdo de nitrogênio no solo.
Portanto, é necessário que sejam desenvolvidos estudos a fim de determinar lâminas de irrigação e níveis de adubação nitrogenada, acompanhada de análise econômica, especificamente para a cultura da alface americana na região sul mineira.

Realizou-se o presente trabalho com o objetivo de estudar o efeito da água, do nitrogênio, e da interação desses fatores sobre os rendimentos produtivo e econômico da cultura da alface-americana irrigada por gotejamento, nas condições edafoclimáticas de Lavras, sul de Minas Gerais.

\section{MATERIAL E MÉTODOS}

O experimento foi conduzido em casa-de-vegetação situada no setor de Olericultura da Universidade Federal de Lavras (UFLA), durante os meses de setembro a novembro de 2004.

O solo da área foi classificado como Latossolo Vermelho distroférrico (EMBRAPA, 1999), textura muito argilosa, e a análise do solo observa-se na Tabela 1.

Utilizou-se neste experimento a alface americana, cultivar Raider. O transplantio foi realizado manualmente em covas espaçadas de $0,30 \times 0,30 \mathrm{~m}$. A grande aceitação desse grupo de alface no Brasil deve-se à sua boa conservação durante o período de armazenamento e, principalmente, sua resistência às altas temperaturas na época de verão, possibilitando o cultivo durante o ano inteiro. As mudas foram doadas por um produtor do município de Alfenas, MG, e foram semeadas no dia 14 de setembro de 2004. Foram cultivadas em bandejas de isopor de 200 células sobre substrato próprio para o cultivo da alface. Decorridos 30 dias da semeadura, ocasião em que as mudas apresentavam por volta de 4 folhas definitivas, efetuou-se o transplantio. $\mathrm{O}$ transplantio foi realizado manualmente em covas espaçadas de $0,30 \times 0,30 \mathrm{~m}$.

O sistema de irrigação utilizado foi o gotejamento, com tubogotejadores NAAN PC, com uma vazão de 1,60 $\mathrm{L} \mathrm{h}^{-1}$, DN $16 \mathrm{~mm}$ e espaçados entre si $0,30 \mathrm{~m}$. Cada parcela experimental possuía duas linhas de gotejadores autocompensantes trabalhando com pressão de serviço de $20 \mathrm{mca}$, sendo que cada linha atendeu a duas linhas de plantas.

Os tratamentos constituíram-se de quatro lâminas de água com base na evaporação do minitanque correspondentes a $35 \%(91,99 \mathrm{~mm}), 70 \%(142,79 \mathrm{~mm}), 100 \%$ $(186,34 \mathrm{~mm})$ e $135 \%(237,14 \mathrm{~mm})$ e quatro doses de nitrogênio com base nas recomendações de Gomes et al. (1999) correspondentes a $0 \%\left(0 \mathrm{~kg} \mathrm{ha}^{-1}\right), 50 \%\left(80 \mathrm{~kg} \mathrm{ha}^{-1}\right)$, $100 \%\left(160 \mathrm{~kg} \mathrm{ha}^{-1}\right)$ e $200 \%\left(320 \mathrm{~kg} \mathrm{ha}^{-1}\right)$.

$\mathrm{O}$ delineamento experimental adotado foi o de blocos casualizados em esquema fatorial $4 \times 4$, com três repetições, perfazendo um total de 48 parcelas. Cada parcela experimental apresentava as dimensões de 1,20 m de largura 
Tabela 1 - Análise química do solo da área experimental*.

\begin{tabular}{lllcc}
\hline Sigla & \multicolumn{1}{c}{ Descrição } & Unidade & \multicolumn{2}{c}{ Amostras** } \\
\cline { 4 - 5 } & & & $0-0,20 \mathrm{~m}$ & $0,20-0,40 \mathrm{~m}$ \\
\hline $\mathrm{pH}$ & em água $(1: 2,5)$ & - & $5,9 \mathrm{AM}$ & $5,6 \mathrm{AM}$ \\
$\mathrm{P}$ & Fósforo (Melich) & $\mathrm{mg} / \mathrm{dm}^{3}$ & $12,4 \mathrm{mb}$ & $3,1 \mathrm{~B}$ \\
$\mathrm{~K}$ & Potássio & $\mathrm{mg} / \mathrm{dm}^{3}$ & $98 \mathrm{~b}$ & $36 \mathrm{~B}$ \\
$\mathrm{Ca}$ & Cálcio & $\mathrm{Cmol} / \mathrm{dm}^{3}$ & $3,7 \mathrm{~b}$ & $2,2 \mathrm{~b}$ \\
$\mathrm{Mg}$ & Magnésio & $\mathrm{Cmol} / \mathrm{dm}^{3}$ & $1,3 \mathrm{~b}$ & $1,1 \mathrm{~b}$ \\
$\mathrm{Al}$ & Alumínio & $\mathrm{Cmol} / \mathrm{dm}^{3}$ & $0,0 \mathrm{MB}$ & $0,2 \mathrm{MB}$ \\
$\mathrm{H}+\mathrm{Al}$ & Ac.potencial & $\mathrm{Cmol} / \mathrm{dm}^{3}$ & $2,1 \mathrm{~B}$ & $2,9 \mathrm{M}$ \\
$\mathrm{SB}$ & Soma bases & $\mathrm{Cmol} / \mathrm{dm}^{3}$ & $5,3 \mathrm{~b}$ & $3,4 \mathrm{M}$ \\
$(\mathrm{t})$ & CTC efetiva & $\mathrm{Cmol} / \mathrm{dm}^{3}$ & $5,3 \mathrm{~b}$ & $3,6 \mathrm{M}$ \\
$(\mathrm{T})$ & CTC a pH 7,0 & $\mathrm{Cmol} / \mathrm{dm}^{3}$ & $7,4 \mathrm{M}$ & $6,3 \mathrm{M}$ \\
$\mathrm{V}$ & Saturação bases & $\%$ & $71,4 \mathrm{~b}$ & $53,9 \mathrm{~b}$ \\
$\mathrm{M}$ & Saturação alumínio & $\%$ & $0 \mathrm{MB}$ & $6 \mathrm{MB}$ \\
$\mathrm{MO}$ & Matéria orgânica & $\mathrm{dag} / \mathrm{kg}$ & $1,2 \mathrm{~B}$ & $1,2 \mathrm{~B}$ \\
$\mathrm{P}$-rem & Fósforo remanescente & $\mathrm{mg} / \mathrm{l}$ & $12,5 \mathrm{~b}$ & $7,5 \mathrm{M}$ \\
\hline
\end{tabular}

* Análise realizada no Laboratório do Departamento de Ciências do Solo. UFLA, Lavras, MG, 2004.

** $\mathrm{A}=$ alto $\mathrm{b}=$ bom $\mathrm{mb}=$ muito bom; $\mathrm{B}=$ baixo $\mathrm{M}=$ médio; $\mathrm{MB}=$ muito baixo; $\mathrm{AM}=$ acidez média

e 2,40 $\mathrm{m}$ de comprimento $\left(2,88 \mathrm{~m}^{2}\right)$. Foram utilizadas quatro linhas de plantas espaçadas de $0,30 \mathrm{~m}$ entre si e $0,30 \mathrm{~m}$ entre plantas, perfazendo um total de 32 plantas por parcela. A área útil da parcela foi constituída pelas duas fileiras centrais $\left(1,08 \mathrm{~m}^{2}\right)$, sendo descartadas a primeira e a última planta dessas linhas, resultando num total de 12 plantas.

A frequiência de irrigação foi diária, sendo a lâmina de irrigação estabelecida de acordo com os tratamentos e baseada na evaporação do minitanque, instalado no centro da casa-de- vegetação. As adubações nitrogenadas foram feitas em 7 aplicações crescentes $(9,11,13,15,16,17$ e $19 \%$ ) sendo a primeira realizada aos 11 dias após o transplantio e a última 3 dias antes da colheita. A adubação com fósforo e potássio foi feita antes do transplantio das mudas para os canteiros.

Desde o transplantio das mudas até o início da diferenciação dos tratamentos (7 dias após o transplantio) foram aplicados $41,18 \mathrm{~mm}$ de água em todos os tratamentos por meio de microaspersores com a finalidade de uniformizar a umidade do solo em todas as parcelas para favorecer o estabelecimento da cultura.

A variável analisada foi a produtividade comercial que foi obtida a partir da pesagem da cabeça comercial. Após obtida a massa fresca comercial e com base nas dimensões das parcelas e considerando-se o espaço entre elas, estimou-se a população de plantas por hectare, encontrando o valor de 78.667 plantas, sendo a produtividade comercial expressa em $\mathrm{kg} \mathrm{ha}^{-1}$.
No estudo da função de produção e das análises econômicas seguiu-se o procedimento adotado por Frizzone (1986).

\section{RESULTADOS E DISCUSSÃO}

A síntese da análise de variância da produtividade comercial encontra-se na Tabela 2 e mostrou efeito significativo da lâmina de água (a $1 \%$ de probabilidade) e sua interação com doses de nitrogênio a $5 \%$ de probabilidade sobre a produtividade comercial da alface americana. Quanto ao efeito do nitrogênio, ele não foi significativo, concordando com Furtado (2001) que também não encontrou efeito significativo da adubação nitrogenada para a massa fresca da parte comercial (produção comercial).

A análise de regressão para lâminas de água (Tabela 2), mostrou efeito altamente significativo, a $1 \%$ de probabilidade, tanto para o modelo linear e o quadrático, porém o segundo modelo apresentou maior coeficiente de determinação $\left(\mathrm{R}^{2}\right)$. Isso mostrou que as lâminas de água aplicadas num intervalo bem amplo foram melhor representadas por uma relação polinomial de segundo grau (equação 1). A equação ajustada (1) apresentou coeficiente de determinação de 0,9848 , evidenciando que mais de $98 \%$ da variação da produção pode ser explicada pelo modelo. De acordo com essa equação, a máxima produtividade, 25.471,93 $\mathrm{kg} \mathrm{ha}^{-1}$, seria obtida com a aplicação de $196,76 \mathrm{~mm}$ de água.

$P C=-1,0419 W^{2}+410,01 W-14865 \quad R^{2}=0,9848$ 
Tabela 2 - Síntese da análise de variância para a produtividade comercial.

\begin{tabular}{lcccc}
\hline Fonte de variação & GL & \multicolumn{3}{c}{ Produtividade comercial $\left(\mathrm{kg} \mathrm{ha}^{-1}\right)$} \\
\cline { 3 - 5 } & & 311244940,28 & $\mathrm{~F}$ & $\mathrm{R}^{2}$ \\
\hline Lâmina de água & 3 & 620294606,76 & $62,415^{* *}$ & \\
Efeito linear & 1 & 299246174,14 & $30,208^{* * *}$ & 0,6643 \\
Efeito quadrático & 1 & 11606224,19 & $1,171^{\mathrm{NS}}$ & 0,9848 \\
Dose nitrogênio & 3 & 14230580,55 & $1,436^{\mathrm{NS}}$ & 0,4087 \\
Efeito linear & 1 & 12867803,12 & $1,299^{\mathrm{NS}}$ & 0,7783 \\
Efeito quadrático & 1 & 22706326,33 & $2,292^{*}$ & \\
Lâmina x dose N & 9 & 11439788,16 & $1,155^{\mathrm{NS}}$ & \\
Bloco & 2 & 9907558,18 & & \\
Resíduo & 30 & & 21401,52 & \\
\hline Média & - & & 14,71 & \\
c.v $(\%)$ & - & & & \\
\hline
\end{tabular}

** e* significativo a $1 \%$ e $5 \%$ de probabilidade, pelo teste $\mathrm{F}$, respectivamente

${ }^{\mathrm{NS}}$ não significativo

Com relação aos valores médios observados da produtividade comercial da alface americana, em função dos tratamentos de lâminas de água e doses de nitrogênio, verificouse que a máxima produtividade da alface americana, $27.841,56 \mathrm{~kg}$ ha $^{-1}$, foi obtida no tratamento $\mathrm{W}_{4} \mathrm{~N}_{3}$, equivalente à aplicação de $237,14 \mathrm{~mm}$ de água e $320 \mathrm{~kg} \mathrm{ha}^{-1}$ de N. Essa combinação é 35\% superior à reposição da evaporação do minitanque e quanto à aplicação de uma dose de nitrogênio, é $100 \%$ superior à recomendada pela análise de solo. $\mathrm{O}$ menor valor médio da produtividade comercial da alface americana, $13.814,25 \mathrm{~kg} \mathrm{ha}^{-1}$, foi obtido no tratamento em que foi aplicada a menor lâmina de água $(91,99 \mathrm{~mm})$. Essa produtividade é inferior àquela obtida no tratamento com dose $0 \mathrm{~kg} \mathrm{ha}^{-1}$ de nitrogênio, cuja produtividade média foi de $19943,7 \mathrm{~kg} \mathrm{ha}^{-1}$, mostrando a importância de uma correta irrigação sobre o rendimento da cultura.

A obtenção das dosagens máximas econômicas de água e nitrogênio, sem que haja a fixação dos níveis de quaisquer dos fatores, leva à construção da superfície de resposta, ou seja, estima-se a produtividade comercial da alface para as duas variáveis: lâmina total de água (W) e dose de nitrogênio $(\mathrm{N})$. A curva de resposta é apresentada pela equação (2), com coeficiente de determinação $\left(\mathrm{R}^{2}\right)$ de 0,8311 . Assim, $83,11 \%$ da variação da produtividade comercial da alface americana são explicadas pela variação de água e dose de nitrogênio.

\section{$\mathrm{PC}=-12490+388,1 \mathrm{~W}-6,02 \mathrm{~N}-1,042 \mathrm{~W}^{2}-0,04563 \mathrm{~N}^{2}+0,1564 \mathrm{WN}$}

em que:

$\mathrm{PC}=$ produtividade comercial da alface americana, em kg ha- ${ }^{-1}$;

$\mathrm{W}=$ lâmina total de água, em mm;

$\mathrm{N}=$ dose de nitrogênio, em $\mathrm{kg} \mathrm{ha}^{-1}$.
Observa-se, na Figura 1, que, nas linhas da superfície, paralelas ao longo dos eixos da água e do nitrogênio, há inicialmente, maior inclinação positiva, que vai diminuindo progressivamente até atingir zero. A partir daí, a inclinação torna-se negativa, indicando redução na produtividade da alface quando os níveis dos fatores excedem certos limites. Observa-se que a água foi o fator mais limitante na produtividade comercial da alface americana, fato esse comprovado pela maior curvatura da linha do fator lâminas totais de água na superfície de resposta e que a resposta da produtividade às doses de nitrogênio foi menos pronunciada.

Conhecendo-se a função de produção (equação 2), chega-se à derivada parcial em relação aos fatores estudados e iguala-se a relação entre o preço do fator correspondente e o preço da alface (equações 3 e 4 ).

$$
\begin{aligned}
& \frac{\partial \mathrm{Y}}{\partial \mathrm{W}}=(388,1-2,084 \mathrm{~W}+0,1564 \mathrm{~N})=\frac{\mathrm{P}_{\mathrm{W}}}{\mathrm{P}_{\mathrm{Y}}} \\
& \frac{\partial Y}{\partial N}=(-6,02-0,09126 N+0,1564 W)=\frac{P_{N}}{P_{Y}}
\end{aligned}
$$

Com os valores conhecidos de $\mathrm{P}_{\mathrm{Y}}, \mathrm{P}_{\mathrm{W}}$ e $\mathrm{P}_{\mathrm{N}}$, estimou-se o valor do fator $\mathrm{N}\left(257,14 \mathrm{~kg} \mathrm{ha}^{-1}\right)$ e do fator $\mathrm{W}(205,26 \mathrm{~mm})$, que correspondem às doses de nitrogênio e de água que proporcionaram a produtividade ótima econômica da alface americana, equivalente a 26.959,93 $\mathrm{kg} \mathrm{ha}^{-1}$, valores abaixo dos obtidos para a produtividade física máxima $\left(27.004,49 \mathrm{~kg} \mathrm{ha}^{-1}\right)$, com lâmina total de água de 208,03 mm e dosagem de $290,50 \mathrm{~kg}$ $\mathrm{ha}^{-1}$ de nitrogênio. Essa lâmina total de água $(208,03 \mathrm{~mm})$ é muito próxima à estimada economicamente no cultivo da alface (205,26 mm), isso porque os custos dos fatores de produção, principalmente a energia gasta para o bombeamento de água, ao longo do ciclo, foram relativamente baixos. 


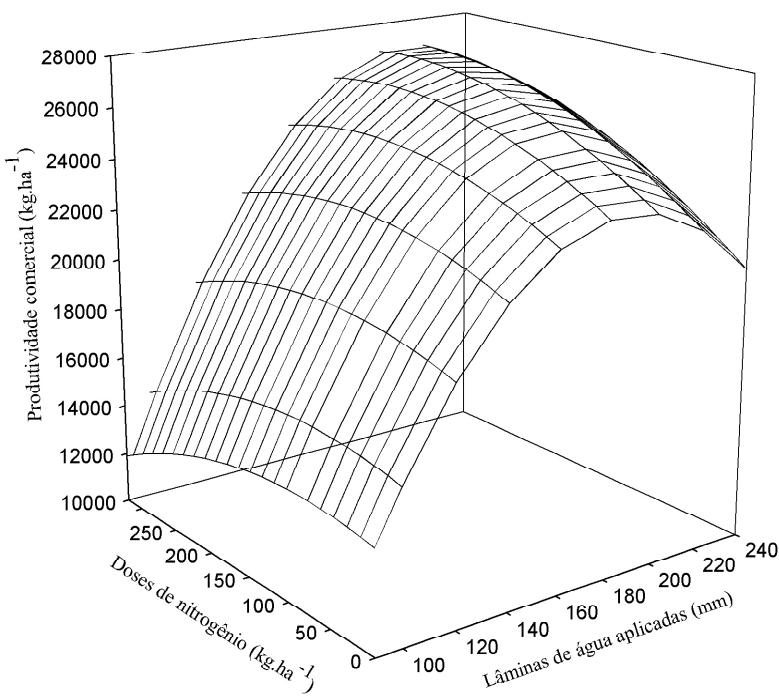

Figura 1 - Produtividade comercial da alface americana, em função das lâminas totais de água e das doses de nitrogênio.

\section{CONCLUSÕES}

Com base nos resultados observados nesse trabalho, conclui-se que:

- a aplicação de 205,26 mm de água e de $257,14 \mathrm{~kg} \mathrm{ha}^{-1}$ de nitrogênio proporcionou estimar produtividade máxima de $26.959,93 \mathrm{~kg} \mathrm{ha}^{-1}$ que correspondeu à obtenção do lucro máximo, considerando os preços dos fatores água $(\mathrm{R} \$ 0,44$ $\left.\mathrm{mm}^{-1}\right)$, nitrogênio $\left(\mathrm{R} \$ 2,09 \mathrm{~kg}^{-1}\right)$ e da alface americana $(\mathrm{R} \$$ $\left.0,80 \mathrm{~kg}^{-1}\right)$;

- a produtividade física máxima de $27.004,49 \mathrm{~kg} \mathrm{ha}^{-1}$ foi estimada com $208,03 \mathrm{~mm}$ e $290,5 \mathrm{~kg} \mathrm{ha}^{-1}$ de nitrogênio;

\section{AGRADECIMENTOS}

À FAPEMIG (Fundação de Amparo à Pesquisa do Estado de Minas Gerais) pelo apoio financeiro dado à pesquisa (Processo № EDT 2232/03).

\section{REFERÊNCIAS BIBLIOGRÁFICAS}

ANDRADE JÚNIOR, A. S. de; RODRIGUES, B. H. N.; ALVES SOBRINHO, C.; MELO, F. de B.; BASTOS, E. A.; CARDOSO, M. J.; RIBEIRO, V. Q. Influência de níveis de irrigação sobre a produtividade e qualidade de frutos de melancia. In: CONGRESSO BRASILEIRO DE ENGENHARIA AGRÍCOLA, 25., 1996, Bauru, SP. Anais... Bauru: SBEA, 1996. p. 141.
BEZERRA, F. M. L.; MOURÃO, R. M. B. Produtividade e qualidade de frutos de melão em função de diferentes níveis de irrigação. In: CONGRESSO BRASILEIRO DE OLERICULTURA, 40., 2000, Brasília, DF. Anais... Brasília, DF: SOB, 2000. p. 601.

EMPRESA BRASILEIRA DE PESQUISA AGROPECUÁRIA. Sistema brasileiro de classificação de solos. Brasília, DF, 1999. 412 p.

FRIZZONE, J. A. Funções de resposta do feijoeiro (Phaseolus vulgaris L.) ao uso de nitrogênio e lâmina de irrigação. 1986. 133 f. Tese (Doutorado em Solos e Nutrição de Plantas) - Escola Superior de Agricultura Luiz de Queiroz, Piracicaba, 1986.

FURTADO, S. C. Nitrogênio e fósforo na produção e nutrição mineral da alface americana cultivada em sucessão ao feijão após o pousio da área. 2001. 78 p. Dissertação (Mestrado em Fitotecnia) - Universidade Federal de Lavras, Lavras, 2001.

GOMES, L. A. A.; SILVA, E. C. da; FAQUIN, V. Recomendações de adubação para cultivos em ambiente protegido. In: RIBEIRO, A. C.; GUIMARÃES, P. T. G.; ALVAREZ, V. H. (Eds.). Recomendações para o uso de corretivos e fertilizantes em Minas Gerais: 5a aproximação. Viçosa: UFV, 1999. p. 99-110.

MAROUELLI, W. A.; PEREIRA, W.; SILVA, W. L. C.; SILVA, H. R. Resposta da aboboreira híbrida a diferentes lâminas de água e doses de nitrogênio. In: CONGRESSO BRASILEIRO DE OLERICULTURA, 40., 2000, Brasília, DF. Anais... Brasília, DF: SOB, 2000. p. 609.

MEDEIROS, J. F.; NASCIMENTO, I. B. do; COSTA, M. da C.; SCALOPPI, E. J. Produção de melão sob diferentes lâminas de água com dois níveis de salinidade. In: CONGRESSO BRASILEIRO DE OLERICULTURA, 40., 2000, Brasília, DF. Anais... Brasília, DF: SOB, 2000. p. 612.

PEREIRA, A. L.; CRUZ, R. L.; KROLL, L. B. Efeitos de lâminas de água e doses de adubação nitrogenada em cobertura, sobre a produção do feijoeiro. In: CONGRESSO BRASILEIRO DE ENGENHARIA AGRÍCOLA, 29., 2000. Fortaleza, CE. Anais... Fortaleza: SBEA, 2000. CD-ROM. 
PEREIRA, O. C. N.; BERTONHA, A.; FREITAS, P. S. L.; GONÇALVES, A. C. A.; REZENDE, R.; SILVA, F. F. da Produção de alface em função de água e de nitrogênio. Acta Scientiarum Agronomy, Maringá, v. 25, n. 2, p. 381-386, 2003.

REICHARDT, K. A água na produção agrícola. São Paulo: McGraw-Hill do Brasil, 1978.
SOARES, J. I.; COSTA, R. N. T.; SILVA, L. A. C.; GONDIM, R. S. Função de resposta da melancia aos níveis de água e adubação nitrogenada, no Vale do Curu, CE. Revista Brasileira de Engenharia Agrícola e Ambiental, Campina Grande, v. 6, n. 2, p. 219-224, jul./ ago. 2002. 